\title{
Characteristics of Progressive Damage of ZnO Nanowires during Contact Sliding under Relatively Low Loads
}

\author{
Kyeong Hee Kang, Hae Jin Kim, and Dae-Eun Kim \\ Department of Mechanical Engineering, Yonsei University 50 Yonsei-ro, Seodaemun-gu, Seoul 120-749, Republic of Korea \\ Correspondence should be addressed to Dae-Eun Kim, kimde@yonsei.ac.kr
}

Received 16 July 2011; Accepted 2 August 2011

Academic Editor: Renzhi Ma

Copyright ( $) 2011$ Kyeong Hee Kang et al. This is an open access article distributed under the Creative Commons Attribution License, which permits unrestricted use, distribution, and reproduction in any medium, provided the original work is properly cited.

$\mathrm{ZnO}$ nanowires have received much interest owing to their particular structural and piezoelectric properties. For widespread application of $\mathrm{ZnO}$ nanowires in various nanotechnologies, the mechanical reliability of the nanowires should be assessed. In this paper, the damage characteristics of vertically grown $\mathrm{ZnO}$ nanowires due to contact sliding against a $2 \mathrm{~mm}$ diameter steel ball under relatively low loads were investigated. Frictional behavior and wear characteristics of the specimens were assessed. Furthermore, contact sliding tests were performed inside an SEM to monitor the progression of damage of the nanowires. It was found that the friction coefficient was about 0.35 under all loads while the damage characteristics of the nanowires were quite different for each load. The large diameter nanowires tended to fracture earlier than the small diameter nanowires. Wear tests performed inside the SEM confirmed the surface damage characteristics observed during the friction tests.

\section{Introduction}

$\mathrm{ZnO}$ nanowires have been receiving much interest as a novel material due to its semiconducting and piezoelectric properties [1-3]. $\mathrm{ZnO}$ nanowires are frequently used as a structural material for the development of nanogenerators and nanosensors. Recently, $\mathrm{ZnO}$ nanowires have been commercially developed as a nanoenergy harvesting material from surrounding energy sources such as noise and vibration [4-7]. $\mathrm{ZnO}$ nanowires often experience contact against a counter surface which can be a packaging material for example. Also, in certain cases, the nanowires may be completely exposed to the atmosphere which can lead to direct contact against other objects. In such cases, the nanowires are susceptible to damage due to mechanical stress caused by intended or unintended contact events. In order to assess the durability or reliability of $\mathrm{ZnO}$ nanowires against mechanical stresses imparted by contact, it is necessary to understand the damage characteristics of the nanowires as they experience contact against a counter surface. Furthermore, since shear stresses are commonly generated at contact points accompanied by a lateral motion, the frictional behavior of the nanowires needs to be investigated as well $[8,9]$.
In this paper, the contact damage characteristics of $\mathrm{ZnO}$ nanowires grown vertically on a silicon substrate were investigated. The progression of nanowire damage was monitored by performing contact sliding experiments using a pin-onreciprocator type tribotester as well as using a wear tester mounted inside a scanning electron microscope (SEM). The frictional behavior of the $\mathrm{ZnO}$ nanowires was also investigated by sliding the nanowires against a steel ball under loads ranging from 2 to $6 \mathrm{mN}$. The motivation was to identify the contact conditions under which $\mathrm{ZnO}$ nanowires get damaged and to understand the damage mechanism. The details of the experiments are described in the following sections.

\section{Experimental Details}

2.1. ZnO Nanowires Synthesis. ZnO nanowires were synthesized on (100) Si wafer with using the hydrothermal method. Prior to the coating processes, Si wafer went through an intensive cleaning process to effectively remove all the organic residues. Si wafer was rinsed with warm $\left(>55^{\circ} \mathrm{C}\right.$ ) acetone and methanol and cleaned by RCA number 1 method using 27\% ammonium hydroxide $\left(\mathrm{NH}_{4} \mathrm{OH}\right)$ and $30 \%$ hydrogen peroxide $\left(\mathrm{H}_{2} \mathrm{O}_{2}\right)$. Si wafers were then dipped 


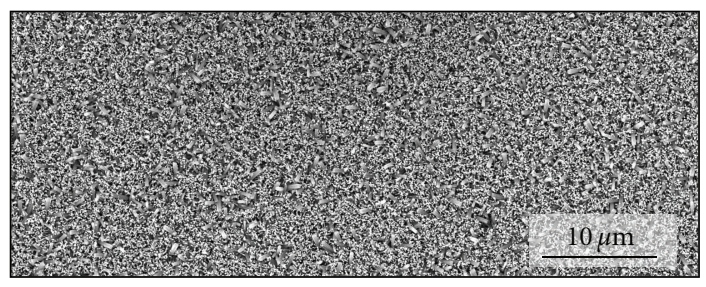

(a)

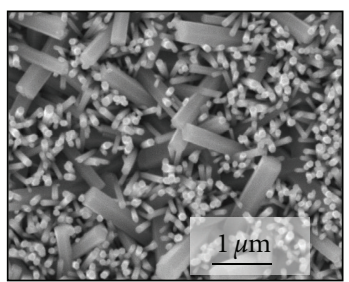

(b)

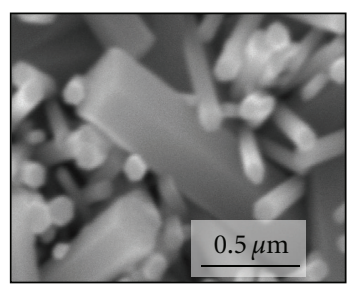

(c)
Figure 1: SEM images of $\mathrm{ZnO}$ nanowires (a) evenly coated on $\mathrm{Si}$ wafer and (b, c) at higher magnification that shows a mixture of nanowires with two distinct diameters.

into hydrogen fluoride (HF) to remove the native silicon dioxide $\left(\mathrm{SiO}_{2}\right)$ layer $[10,11]$. Additionally, they were exposed under ultraviolet-ozone (UVO) light for $20 \mathrm{~min}$ to increase the adhesion property of the substrate surface.

The specimen fabrication process consisted of two steps: (1) $\mathrm{ZnO}$ seed-layer coating and (2) growth of $\mathrm{ZnO}$ nanowire arrays. For $\mathrm{ZnO}$ seed layer coating, a solution of $20 \mathrm{mM}$ EtOH and Zinc acetate dihydrate $\left(\left(\mathrm{C}_{2} \mathrm{H}_{3} \mathrm{O}_{2}\right)_{2} \mathrm{Zn} \cdot 2 \mathrm{H}_{2} \mathrm{O}\right)$ were aged for more than 6 hours. The process of spin coating was repeated several times to attain adequate thickness. Si wafer with the seed layer was immersed in the solution of HPLC (high-performance liquid chromatography) grade deionized water, zinc nitrate hexahydrate $\left(\mathrm{Zn}\left(\mathrm{NO}_{3}\right)_{2}\right)$, and hexamethylene-tetramine (HMT, $\mathrm{C}_{6} \mathrm{H}_{12} \mathrm{~N}_{4}$ ) at $80^{\circ} \mathrm{C}$ condition for 6 hours [12-14]. Finally, $\mathrm{ZnO}$ nanowires on Si wafers were carefully rinsed with deionized water and dried at room temperature. The main chemical process can be described as (1) $[15]$.

$$
\begin{gathered}
\left(\mathrm{CN}_{2}\right)_{6} \mathrm{~N}_{4}+6 \mathrm{H}_{2} \mathrm{O} \longrightarrow 6 \mathrm{HCHO}+4 \mathrm{NH}_{3} \\
\mathrm{NH}_{3}+\mathrm{H}_{2} \mathrm{O} \longrightarrow \mathrm{NH}_{4}^{+}+\mathrm{OH}^{-} \\
\left(2 \mathrm{OH}^{-}+\mathrm{Zn}^{2+} \longrightarrow \mathrm{Zn}(\mathrm{OH})_{2}\right) \\
\mathrm{Zn}(\mathrm{OH})_{2} \longrightarrow \mathrm{ZNO}+\mathrm{H}_{2} \mathrm{O}
\end{gathered}
$$

The morphology of the $\mathrm{ZnO}$ nanowires coated by the above described method is shown in SEM images of Figure 1. Figure 1(a) shows that the nanowires are well distributed evenly over the entire area. High magnification images of Figures 1(b) and 1(c) show that the nanowires are vertically aligned on the average but not perfectly. Also, it can be seen that there are two distinct sizes in the nanowires grown on the silicon substrate. The majority of the nanowires have a diameter of less than $120 \mathrm{~nm}$. However, mixed with these nanowires, those with a diameter of about $350 \mathrm{~nm}$ can be found. It was also found that the height of the smaller diameter nanowires was about $1.2 \mu \mathrm{m}$ while some of the

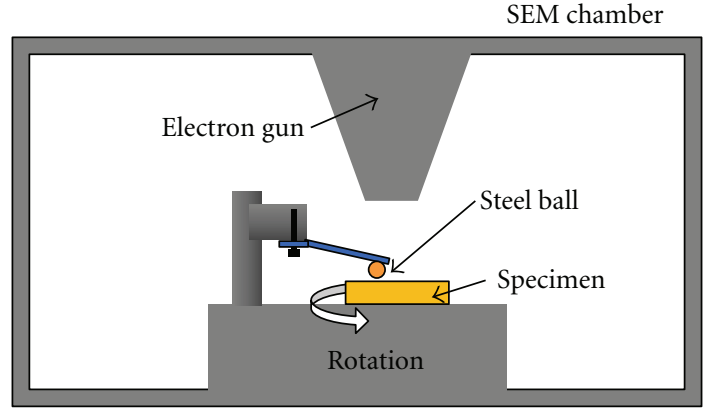

FIGURE 2: Schematic of the pin-on-disk type wear tester mounted inside the SEM.

larger diameter nanowires were about 0.1 to $0.3 \mu \mathrm{m}$ longer. The aspect ratio of the large and small diameter nanowires was about 4 and 10, respectively.

2.2. Friction Test. Contact sliding tests were performed using a pin-on-reciprocator type tribotester to investigate the frictional behavior of the $\mathrm{ZnO}$ nanowires. Frictional force was monitored in real time while reciprocating a $2 \mathrm{~mm}$ diameter steel ball in contact with the $\mathrm{ZnO}$ nanowire specimen surface. The tests were performed for 20 seconds with a linear speed of $4 \mathrm{~mm} / \mathrm{sec}$ under the normal loads of 2,4 , and $6 \mathrm{mN}$. The friction tests were performed five times under the same condition. After each test, the steel ball was carefully cleaned with acetone, $\mathrm{EtOH}$, and de-ionized water to minimize the effect of ball contamination. The wear of the steel ball was found to be insignificant after each test. Thus, the same ball could be used for all the friction tests after cleaning. The wear tracks formed on the $\mathrm{ZnO}$ nanowire specimen during the friction tests were observed using the SEM.

2.3. Wear Test Inside an SEM. In order to assess the progression of $\mathrm{ZnO}$ nanowire damage due to contact sliding without interruption of the sliding motion wear tests were performed inside an SEM. A pin-on-disk type wear tester was mounted inside the SEM to observe the wear behavior of $\mathrm{ZnO}$ nanowires in real time. Figure 2 shows the schematic of the wear tester system used in this paper. The normal load was applied using a $z$-axis microstage based on premeasured load-displacement calibration data. The tests were performed at the rotational speed of 2 RPM for 10 cycles under the normal loads of 2, 4, and $6 \mathrm{mN}$ in vacuum condition $\left(10^{-3} \mathrm{~Pa}\right)$. A $2 \mathrm{~mm}$ steel ball was used for the counter surface and cleaned after each experiment with acetone, $\mathrm{EtOH}$, and de-ionized water to minimize contamination effects. The wear track was observed at the 5 th and the 10th cycles to characterize the contact damage of $\mathrm{ZnO}$ nanowires for each load.

\section{Tribological Characteristics of ZnO Nanowires}

3.1. Results of the Friction Tests. The contact sliding tests were conducted using the $\mathrm{ZnO}$ nanowire specimens under various loads while obtaining the frictional force in real time. 


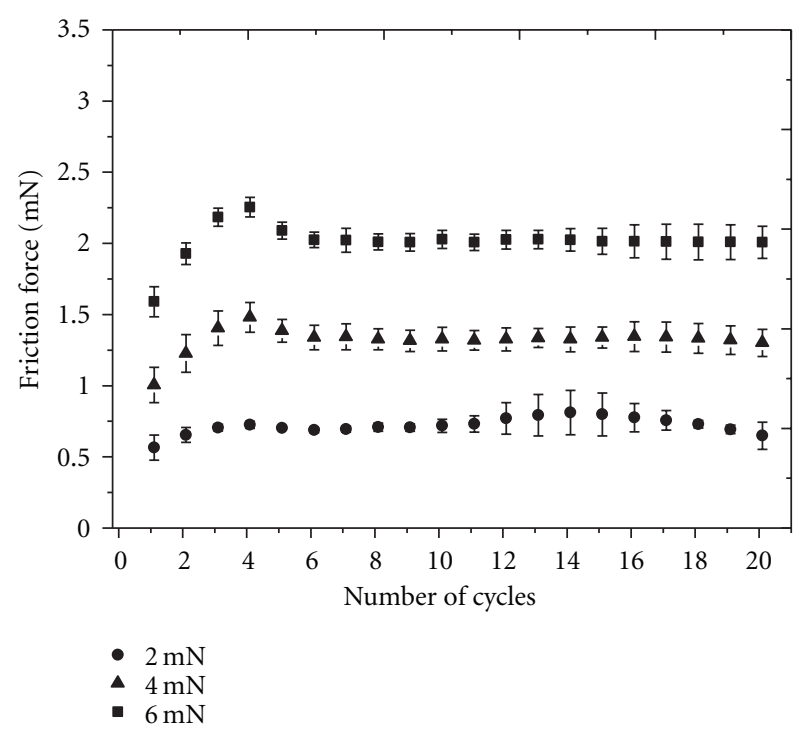

FIgURE 3: Frictional force of $\mathrm{ZnO}$ nanowires sliding against a $2 \mathrm{~mm}$ diameter steel ball with respect to number of sliding cycles for different applied loads.

Figure 3 shows the frictional behavior of $\mathrm{ZnO}$ nanowires with respect to the number of cycles for the normal loads of 2, 4, and $6 \mathrm{mN}$. The frictional forces were averaged over five repeated tests and the standard deviations were also calculated. It can be seen that for all cases the frictional force gradually increased to a steady state value after a few reciprocating cycles. Also, the frictional force increased with the increase in the applied load as expected.

Figure 4 shows the friction coefficients and their standard deviations calculated from the friction data. The friction coefficient during the first cycle for 2, 4, and $6 \mathrm{mN}$ applied load was $0.28,0.25$, and 0.27 , respectively. The values gradually increased with the number of sliding cycles. It was found that the steady state friction coefficient was about 0.35 for all three loads. The frictional behaviors of 4 and $6 \mathrm{mN}$ applied load cases were almost identical. For the $2 \mathrm{mN}$ case, there was an interval in the number of sliding cycles where the friction coefficient increased to about 0.4 and then decreased to about 0.35 . The explanation for this behavior is not straightforward. A reasonable thought is that for the lowest applied load of $2 \mathrm{mN}$, the contact was less stable than for higher applied loads, and therefore, the frictional force was affected more by the disturbances introduced at the sliding interface such as wear particles. This view is supported by the high standard deviation in the friction data in that particular range of sliding cycles for the $2 \mathrm{mN}$ applied load. Nevertheless, for the range of applied load used in this work, it would be acceptable to state that the friction coefficient was similar for all applied loads.

The damage of $\mathrm{ZnO}$ nanowires due to the frictional interaction was assessed by observing the wear tracks using the SEM. Figure 5 shows the SEM images of the wear track of $\mathrm{ZnO}$ nanowires after the friction tests conducted under 2,4 , and $6 \mathrm{mN}$ for $1-20$ cycles. The symbols shown in the SEM images indicate different damage characteristics

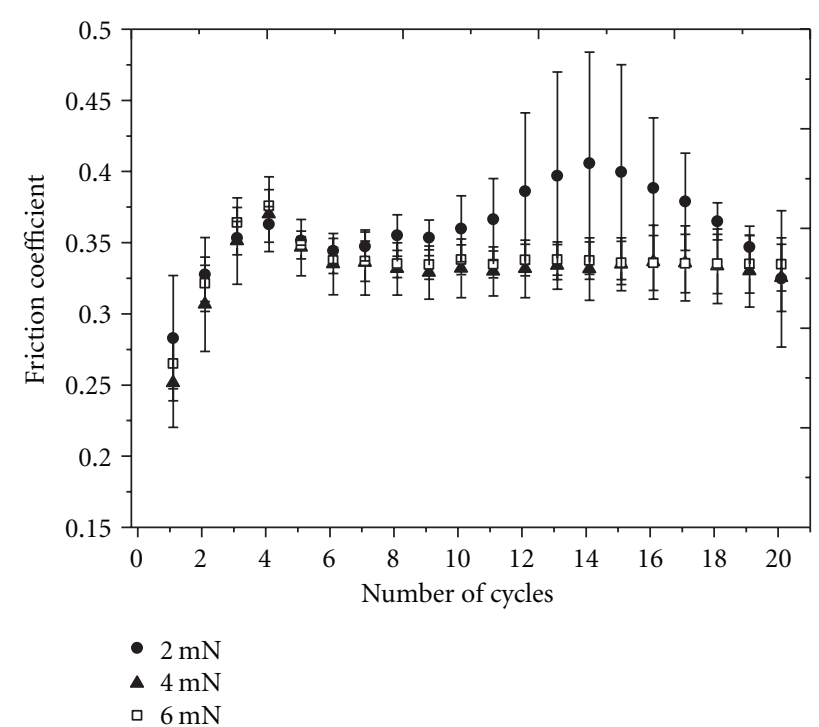

FIGURE 4: Friction coefficient of $\mathrm{ZnO}$ nanowires sliding against a $2 \mathrm{~mm}$ diameter steel ball with respect to number of sliding cycles for different applied loads.

of nanowires. The damage of $\mathrm{ZnO}$ nanowires with large diameter is indicated by a triangle symbol $(\triangleright)$. The damage of $\mathrm{ZnO}$ nanowires with small diameter is specified with an arrow $(\rightarrow)$. Finally, the dotted region shown in the SEM region indicates severe damage and compacted region of $\mathrm{ZnO}$ nanowires. Figure 5(a) shows the SEM images of the wear track of $\mathrm{ZnO}$ nanowires after the friction tests conducted under $2 \mathrm{mN}$ for 10 and 20 cycles of sliding. For number of cycles below 7 , the damage of the $\mathrm{ZnO}$ nanowires was too small to be identified with the SEM. It could be found that after 10 reciprocating cycles of sliding under $2 \mathrm{mN}$, the overall morphology of the $\mathrm{ZnO}$ nanowire specimen seemed to be intact. It was interesting to note that there were some fractured fragments of large diameter nanowire as indicated by the arrows in the figure. Less number of fractured small diameter nanowires could be found. This suggested that the large diameter nanowire tended to fail more readily than the small diameter nanowire when a mechanical stress was applied through contact sliding. After 20 cycles of sliding the number of fractured large diameter nanowires increased, but the damage of the small diameter nanowires was still not severe.

Under the $4 \mathrm{mN}$ applied load, the damage of $\mathrm{ZnO}$ nanowires with large diameter occurred within the first cycle. The number of fractured $\mathrm{ZnO}$ nanowires with large diameter was slightly increased after 3 cycles. After 5 cycles, large diameter $\mathrm{ZnO}$ nanowires were damaged more severely. Small diameter $\mathrm{ZnO}$ nanowires also started to fracture into smaller pieces as the number of cycles increased. After 10 cycles under $4 \mathrm{mN}$ applied load, the fractured $\mathrm{ZnO}$ nanowires were compressed to form a compacted surface morphology due to repetitive and relatively high contact stresses. After 20 cycles, it could be found that the extent of compressed fractured $\mathrm{ZnO}$ nanowires increased significantly (Figure 5(b)). As evident from Figure 5(c), the damage characteristics under 


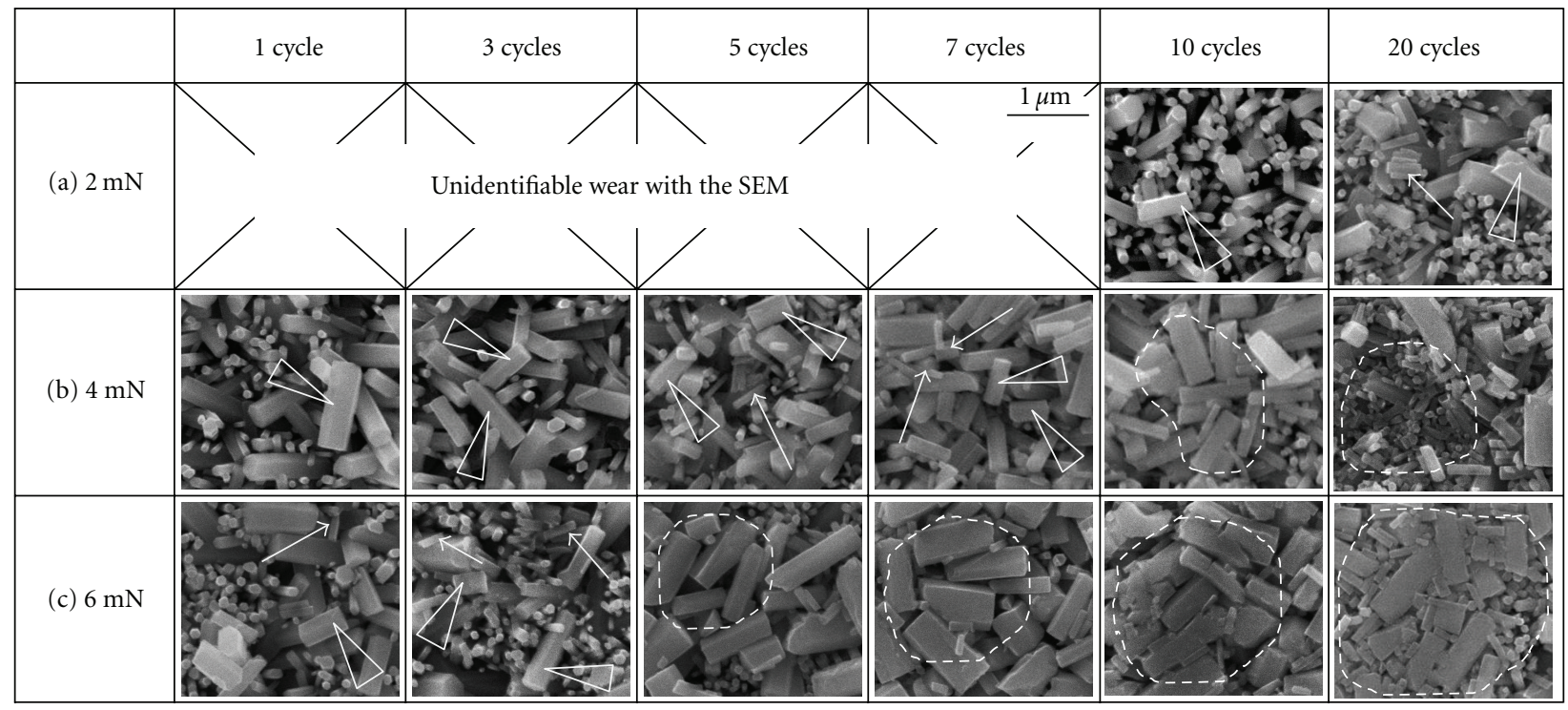

Figure 5: SEM image of the wear track on the $\mathrm{ZnO}$ nanowire specimen after the friction test under (a) $2 \mathrm{mN}$, (b) $4 \mathrm{mN}$, and (c) $6 \mathrm{mN}$ applied load after 1-20 cycles of contact sliding. ( $\triangleright$ : fracture of $\mathrm{ZnO}$ nanowire with large diameter, $\rightarrow$ : fracture of $\mathrm{ZnO}$ nanowire with small diameter, dotted regions: the severe damage and compacted region of $\mathrm{ZnO}$ nanowires).

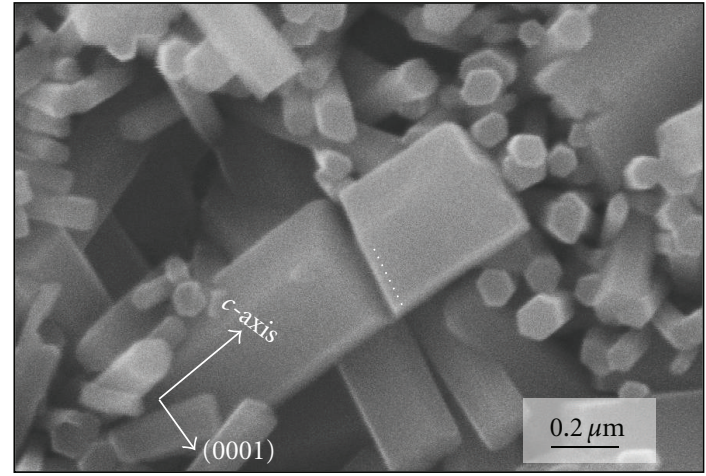

(a)

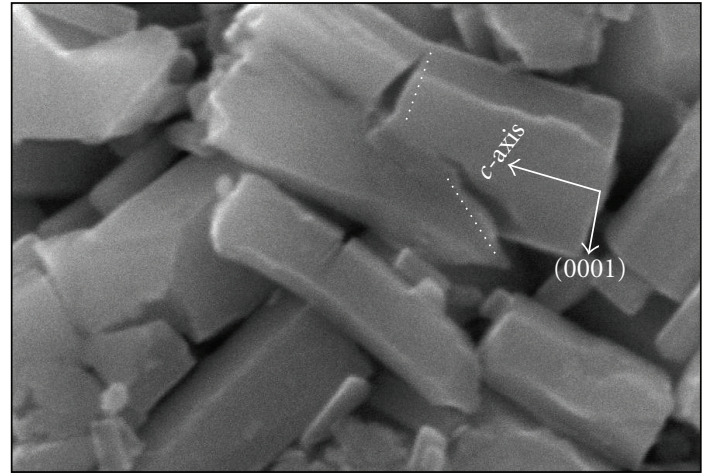

(b)

FIGURE 6: SEM image of ZnO nanowire fractured in (a) parallel to the (0001) plane after 3 cycles under $6 \mathrm{mN}$ applied load and (b) both parallel and nonparallel to the (0001) plane after 10 cycles under $6 \mathrm{mN}$ applied load. Dotted lines show the fractured plane of the ZnO nanowires. (0001) is the perpendicular plane to the $c$-axis of $\mathrm{ZnO}$ crystal that represents the growing direction of $\mathrm{ZnO}$ nanowires.

$6 \mathrm{mN}$ applied load was similar to the cases of 2 and $4 \mathrm{mN}$ applied loads. However, the damage progressed at a faster rate at the higher load. The fracture of $\mathrm{ZnO}$ nanowires with both small and large diameter initiated within the first cycle. Patches of compressed region of fractured $\mathrm{ZnO}$ nanowires were found along the wear track after 5 cycles. The coverage area of the compressed region on the wear track continuously expanded with the increasing number of cycles. Overall, it would be reasonable to assert that the progression characteristics of damage of $\mathrm{ZnO}$ nanowires were similar for all applied loads tested in this work.

It was also interesting to observe the damage behavior of $\mathrm{ZnO}$ nanowires with the number of sliding cycles. Figure 6 shows the magnified image of the fractured nanowires. The fracture direction of a selected $\mathrm{ZnO}$ nanowire was indicated by dotted lines in the figure. The $\mathrm{ZnO}$ nanowire was mostly fractured parallel to the (0001) plane after 3 cycles under $6 \mathrm{mN}$ applied load as shown in Figure 6(a). Fractured $\mathrm{ZnO}$ nanowires with (0001) plane appeared commonly for most of the sliding cycles under all applied loads. $\mathrm{ZnO}$ nanowires that fractured along a different plane was also found after 10 cycles under $6 \mathrm{mN}$ load as shown in Figure 6(b). The fracture of $\mathrm{ZnO}$ nanowire that occurred in a nonparallel plane to the (0001) plane was observed only among the fractured and compressed $\mathrm{ZnO}$ nanowires. However, they were found mostly in wear tests performed at high loads or after a sufficient number of cycles such as 10-20 cycles under $4 \mathrm{mN}$ applied load or 5-20 cycles under $6 \mathrm{mN}$ applied load. Therefore, it was noted that high and repetitive stresses can lead to $\mathrm{ZnO}$ nanowire fracture in a plane that is nonparallel 

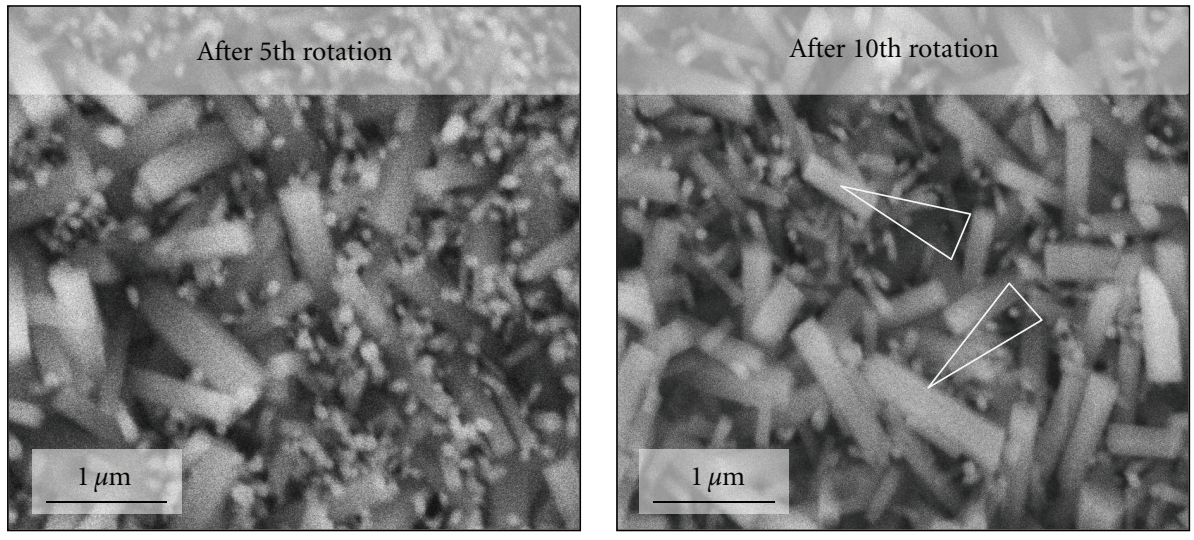

(a)
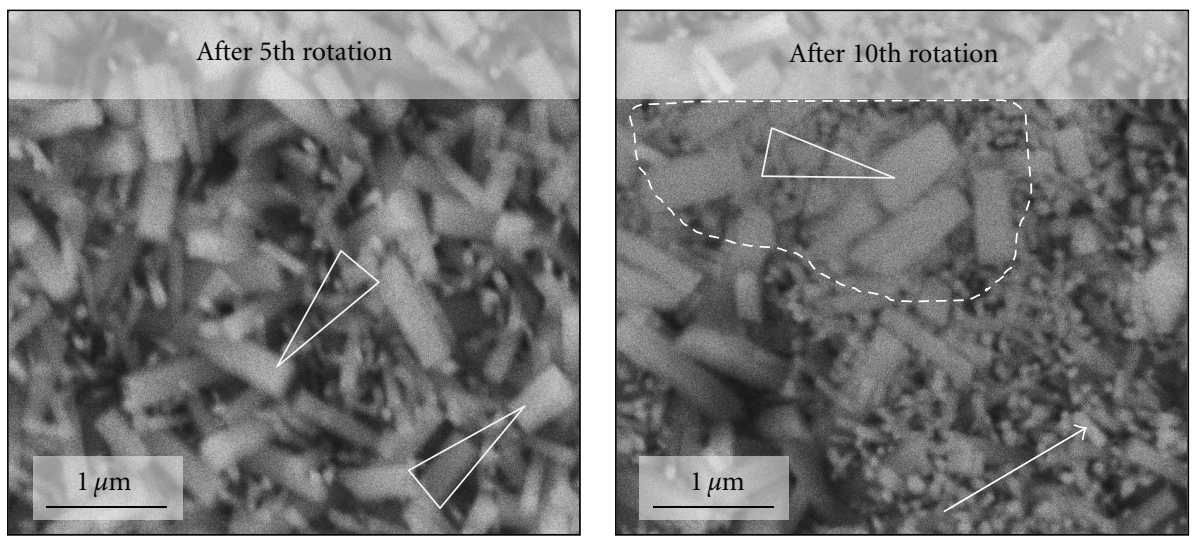

(b)
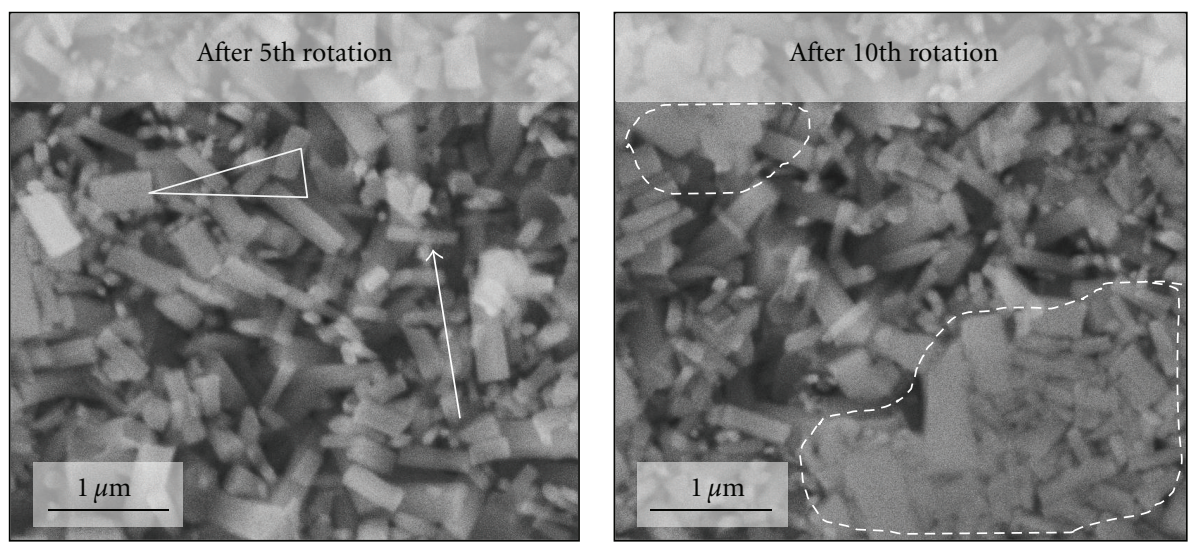

(c)

FIGURE 7: SEM image of the wear test under the load of (a) $2 \mathrm{mN}$, (b) $4 \mathrm{mN}$, and (c) $6 \mathrm{mN}$ performed inside the SEM. Inset of triangle, arrow, and dotted regions show the large diameter, small diameter and compressed region of fractured $\mathrm{ZnO}$ nanowires, respectively.

to the (0001) plane. The different fracture behavior of $\mathrm{ZnO}$ nanowires with respect to the fracture plane was also reported in previous researches $[16,17]$.

3.2. Results of the Wear Test Inside an SEM. In order to investigate the progression of damage of $\mathrm{ZnO}$ nanowires during repeated contact sliding, wear tests were performed inside the SEM. Figure 7 shows the SEM images within the wear tracks of the $\mathrm{ZnO}$ nanowire specimens after sliding against the steel ball under three different loads. The surface state of the $\mathrm{ZnO}$ nanowire specimens after 5 and 10 cycles of rotation is presented on the right and on the left sides, respectively. The damage characteristics of the $\mathrm{ZnO}$ nanowires were visually similar to the wear test results obtained from the friction test.

For the $2 \mathrm{mN}$ wear test, the amount of fractured nanowires was minimal after the 5 th rotation while some fracture of the large diameter nanowires could be found after the 10th rotation as shown in Figure 7(a). Under the load of $4 \mathrm{mN}$, 


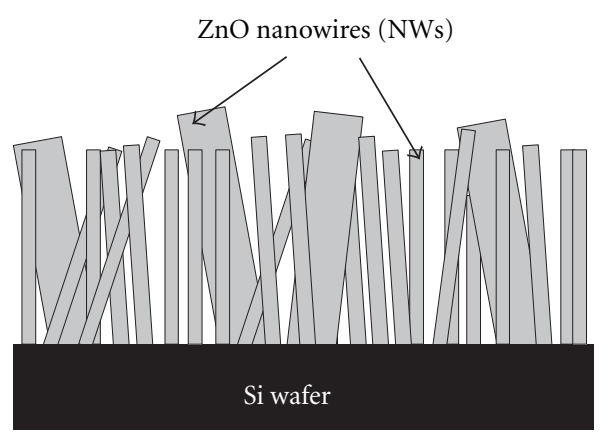

(a)

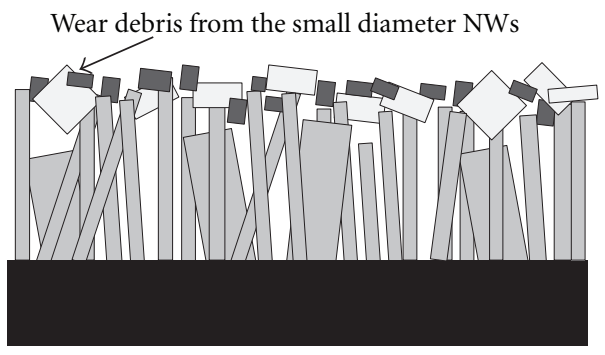

(c)

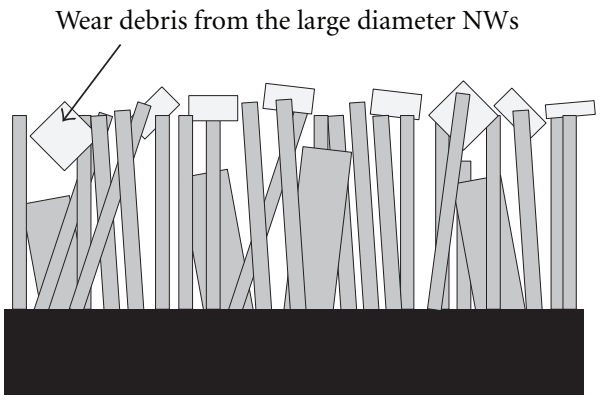

(b)

Compact surface

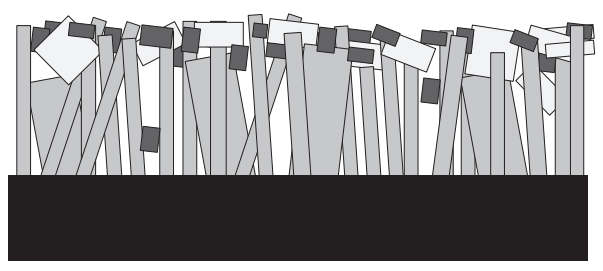

(d)

Figure 8: Illustration of $\mathrm{ZnO}$ nanowire damage process: (a) undamaged $\mathrm{ZnO}$ nanowires, (b) wear debris of fractured fragments of large diameter $\mathrm{ZnO}$ nanowires, (c) wear debris from fractured fragments of small diameter $\mathrm{ZnO}$ nanowires, and (d) compact surface formation due to compression of wear debris through repetitive and high contact stresses.

fracture of the large diameter $\mathrm{ZnO}$ nanowires could be found after the 5th rotation and severe damage was apparent after the 10th rotation as shown in Figure 7(b). Also, regions of fractured nanowires in a compacted form could be found along the wear track just as that observed after the friction tests. Figure 7(c) represents the result of the wear test under $6 \mathrm{mN}$ applied load. $\mathrm{ZnO}$ nanowires were significantly damaged just after the 5th rotation and a large number of fractured nanowires covered the surface of vertically grown $\mathrm{ZnO}$ nanowires. After the 10th rotation, fractured $\mathrm{ZnO}$ nanowires on the surface were pressed down (dotted region) and penetrated into the $\mathrm{ZnO}$ nanowires due to high contact stresses.

\subsection{Mechanism of the Contact Damage of $\mathrm{ZnO}$ Nanowire.} From the results of the contact sliding tests the mechanism of contact damage of $\mathrm{ZnO}$ nanowires was postulated as illustrated in Figure 8. The fractured fragments of the nanowires were distributed on the surface of the nanowires rather than penetrate into the nanowire structure. Fragments of the large diameter nanowires were initially created (Figure 8(a)) followed by those of the small diameter nanowires (Figure $8(\mathrm{~b})$ ). With repeated cycles of sliding the number of fractured fragments of the nanowires increased and got compressed due to the normal load. After a sufficient number of sliding cycles a continuous compact layer of fractured fragments of nanowires was formed on the surface (Figure $8(d)$ ).

Unlike most sliding systems where the surface wears gradually, the damage of $\mathrm{ZnO}$ nanowires due to repeated contact sliding occurred mostly by fracture. Thus, the wear debris created were in the shape of fragmented portions of the nanowires. As mechanical stress was imparted on the nannowires due to contact sliding some of the nanowires experienced fracture along the (0001) plane of the $\mathrm{ZnO}$ crystal. Fracture planes other than (0001) were also found. The extent of damage increased with increasing applied load and number of sliding cycles. At the lowest load of $2 \mathrm{mN}$ used in this work nanowire damage was not evident during the first several cycles of sliding. On the other hand, damage of the nanowires by fracture occurred within the first sliding cycle at loads higher than $4 \mathrm{mN}$.

An interesting point to note in the behavior of nanowire damage was that the large diameter nanowires tended to fail more readily than the small diameter ones. This outcome was attributed to two factors. The first factor is regarding the length of the nanowires. Since the large diameter nanowires were slightly longer than the small diameter nanowires, they could have been fractured first while protecting the small diameter nanowires from being damaged. However, considering the small percentage of nanowires with longer length, this effect was not considered to be significant. The second factor is regarding the stiffness of the nanowires. As mentioned before, the aspect ratio of large diameter nanowires was much smaller than the small diameter nanowires. Thus, high stiffness due to low aspect ratio of the large diameter nanowires could have resulted in early failure. Due to the high stiffness, the large diameter nanowires were not prone to elastic deflection that was needed to dissipate the frictional energy without being plastically deformed. In the case of the small diameter nanowires dissipation of the frictional energy was possible through accumulation of the elastic energy within the nanowire which was then released once the contact event was over. Nevertheless, even the small diameter 
nanowires got damaged by fracture when the load was sufficiently high or the number of contact cycles increased.

\section{Conclusion}

In this paper, the contact damage characteristics and frictional behavior of $\mathrm{ZnO}$ nanowires were investigated. The progression of damage of $\mathrm{ZnO}$ nanowires was investigated through analysis of the wear tracks after each cycle as well as by wear tests performed inside an SEM. Based on the experimental results, the following conclusions may be drawn.

(1) The friction coefficient was about 0.35 for the applied loads of 2, 4, and $6 \mathrm{mN}$ throughout the entire sliding cycles.

(2) Large diameter nanowires tended to fracture more readily than small diameter nanowires during frictional interaction due to higher stiffness.

(3) Gradually, compressed fragments of fractured nanowires covered the surface of vertically grown $\mathrm{ZnO}$ nanowires which resulted in the formation of a continuous compact surface.

(4) The damage characteristics of $\mathrm{ZnO}$ nanowires could be classified into three steps: fracture of $\mathrm{ZnO}$ nanowires with large diameter, fracture of $\mathrm{ZnO}$ nanowires with large and small diameter, and compression of the fractured nanowires to form a compact surface.

\section{Acknowledgment}

This work was supported by the National Research Foundation of Korea (NRF) grant funded by the Korea government (MEST) no. 2011-0000409.

\section{References}

[1] J. H. Ko, K. Jung, S. Kim, W. Kim, and M. Cho, "A proper orthogonal decomposition for parametric study of the mechanical behavior of nanowires," Journal of Mechanical Science and Technology, vol. 25, no. 1, pp. 157-162, 2011.

[2] N. K. Park, G. B. Han, S. H. Yoon, S. O. Ryu, and T. J. Lee, "Preparation and absorption properties of $\mathrm{ZnO}$ nanostructures for cleanup of H2S contained gas," International Journal of Precision Engineering and Manufacturing, vol. 11, no. 2, pp. 321-325, 2010.

[3] Z. L. Wang, "Splendid one-dimensional nanostructures of zinc oxide: a new nanomaterial family for nanotechnology," American Chemical Society Nano, vol. 2, no. 10, pp. 1987-1992, 2008.

[4] W. K. Hong, J. I. Sohn, D. K. Hwang et al., "Tunable electronic transport characteristics of surface architecture-controlled ZnO nanowire field effect transistors," Nano Letters, vol. 8, no. 3, pp. 950-956, 2008.

[5] S. Xu, Y. Qin, C. Xu, Y. Wei, R. Yang, and Z. L. Wang, "Selfpowerednanowire devices," Nature Nanotechnology, vol. 5, pp. 366-373, 2010.

[6] Z. L. Wang and J. Song, "Piezoelectric nanogenerators based on zinc oxide nanowire arrays," Science, vol. 312, no. 5771, pp. 243-246, 2006.
[7] J. I. Hahm and C. M. Lieber, "Direct ultrasensitive electrical detection of DNA and DNA sequence variations using nanowire nanosensors," Nano Letters, vol. 4, no. 1, pp. 51-54, 2004.

[8] L. Y. Lin, J. M. Seo, M. C. Jeong, K. J. Koo, D. E. Kim, and J. M. Myoung, "Wear rate of vertically grown $\mathrm{ZnO}$ nanowires sliding against steel micro-sphere," Materials Science and Engineering A, vol. 460-461, no. 5, pp. 370-376, 2007.

[9] K. H. Chung, H. J. Kim, L. Y. Lin, and D. E. Kim, "Tribological characteristics of $\mathrm{ZnO}$ nanowires investigated by atomic force microscope," Applied Physics A, vol. 92, no. 2, pp. 267-274, 2008.

[10] E. M. Arnett, Prudent Practices in the Laboratory, National Academy Press, 1995.

[11] W. Kern, Handbook of Semiconductor Cleaning Technology, William Andrew Publishing/Noyes, 1993.

[12] L. Vayssieres, "Growth of arrayed nanorods and nanowires of $\mathrm{ZnO}$ from aqueous solutions," Advanced Materials, vol. 15, no. 5, pp. 464-466, 2003.

[13] M. Wang, C. H. Ye, Y. Zhang et al., "Synthesis of well-aligned $\mathrm{ZnO}$ nanorod arrays with high optical property via a lowtemperature solution method," Journal of Crystal Growth, vol. 291, no. 2, pp. 334-339, 2006.

[14] L. W. Ji, S. M. Peng, J. S. Wu, W. S. Shih, C. Z. Wu, and I. T. Tang, "Effect of seed layer on the growth of well-aligned $\mathrm{ZnO}$ nanowires," Journal of Physics and Chemistry of Solids, vol. 70, no. 10, pp. 1359-1362, 2009.

[15] Q. Ahsanulhaq, A. Umar, and Y. B. Hahn, "Growth of aligned $\mathrm{ZnO}$ nanorods and nanopencils on $\mathrm{ZnO} / \mathrm{Si}$ in aqueous solution: growth mechanism and structural and optical properties," Nanotechnology, vol. 18, no. 11, Article ID 115603, 2007.

[16] S. Hoffmann, F. Östlund, J. Michler et al., "Fracture strength and Young's modulus of $\mathrm{ZnO}$ nanowires," Nanotechnology, vol. 18, no. 20, Article ID 205503, 2007.

[17] F. Xu, Q. Qin, A. Mishra, Y. Gu, and Y. Zhu, "Mechanical properties of $\mathrm{ZnO}$ nanowires under different loading modes," Nano Research, vol. 3, no. 4, pp. 271-280, 2010. 

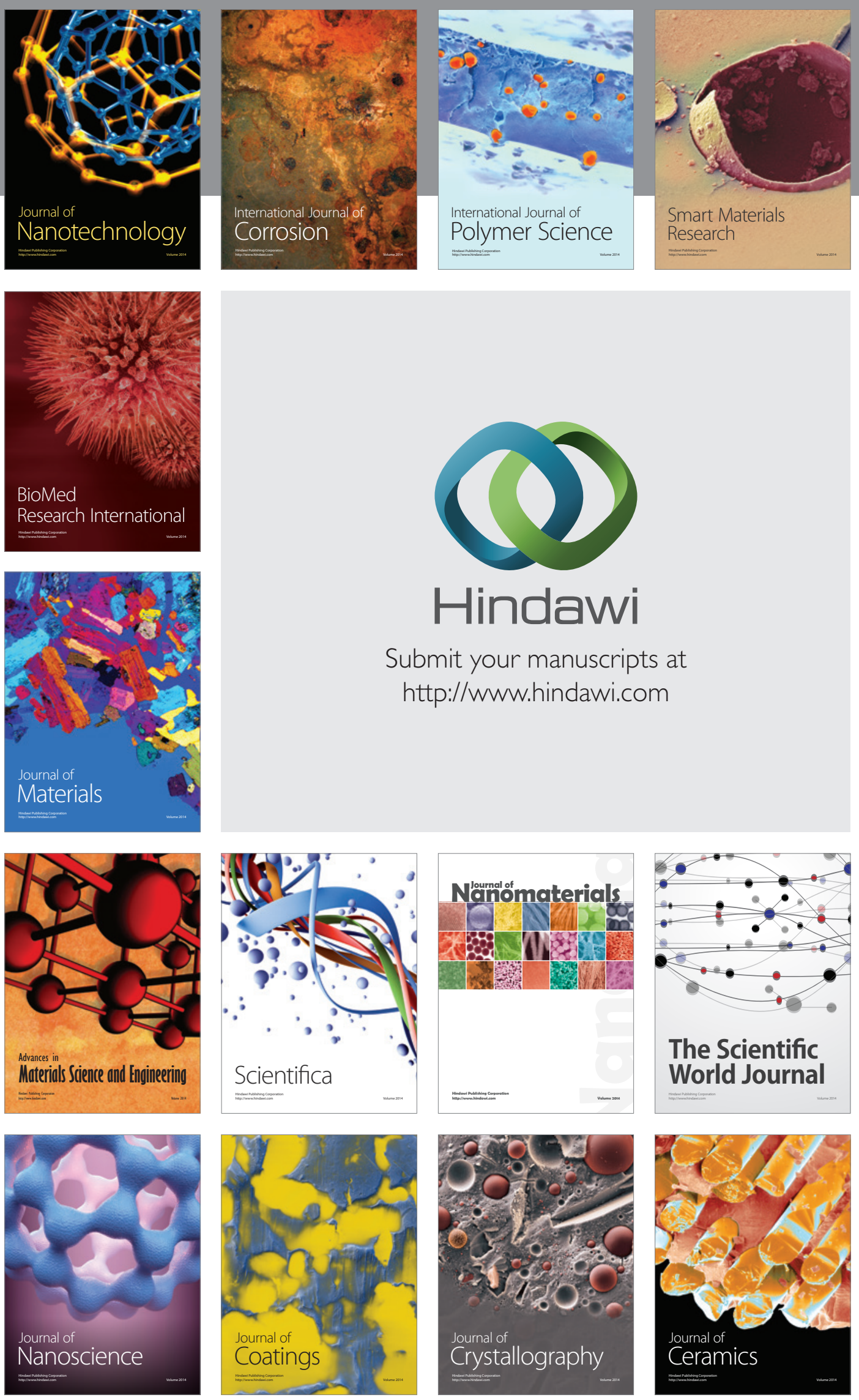

The Scientific World Journal

Submit your manuscripts at

http://www.hindawi.com

\section{World Journal}

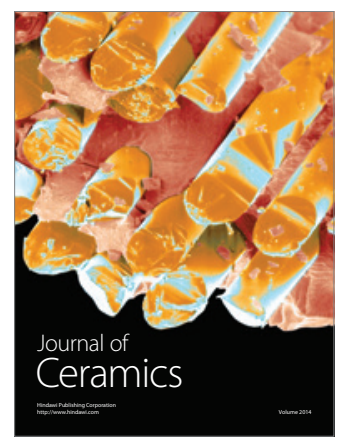

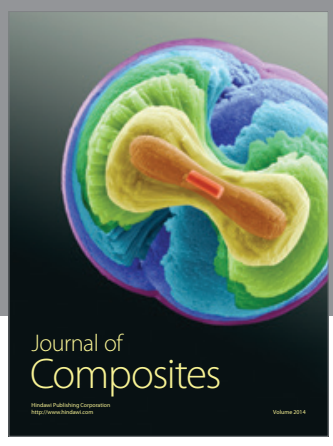
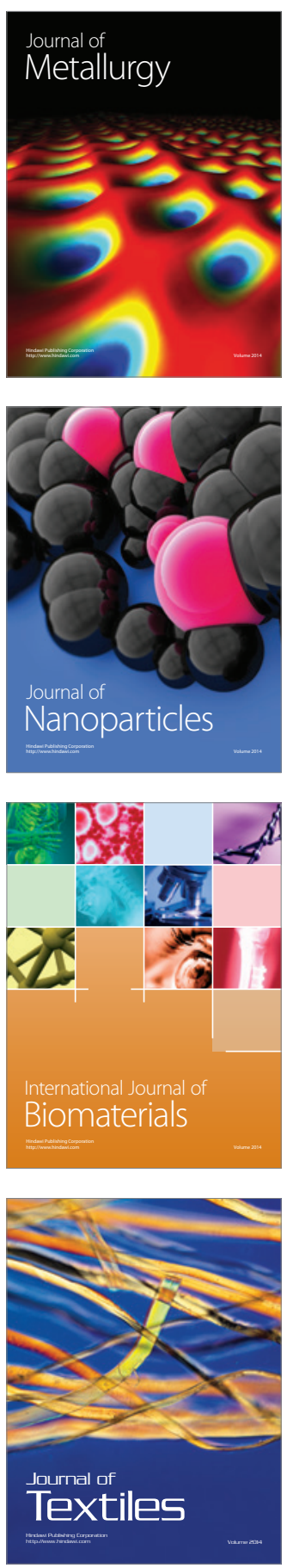